\title{
Urinary Urgency, CTCAE
}

National Cancer Institute

\section{Source}

National Cancer Institute. Urinary Urgency, CT CAE. NCI Thesaurus. Code C143907.

A disorder characterized by a sudden compelling urge to urinate. 